\title{
Aplicação de FT-MIR e FT-NIR ao Estudo de Reação de Cura de Sistemas Epoxídicos
}

\author{
Benedita M. V. Romão \\ Instituto Tecnológico da Aeronáutica, CTA \\ Milton F. Diniz, Luiz C. Pardini, Rita C. L. Dutra \\ Instituto de Aeronáutica e Espaço, CTA
}

Resumo: A reação de cura entre amostras de resina epoxídica (EP) e compostos à base de mercaptana (SH), amino-fenol e amina modificada foi estudada nas regiões espectrais do infravermelho médio (MIR) e próximo (NIR). Observou-se, basicamente, que a espectroscopia FT-NIR evidencia melhor as alterações espectrométricas ocorridas durante as reações estudadas, permitindo detectar, inclusive, o agente de cura em menor proporção no sistema epoxídico.

Palavras-chave: Resina epoxídica, agentes de cura, mercaptana, amino-fenol. amina modificada, alterações espectrométricas, MIR, NIR.

\section{FT-IR MIR and FT-NIR Applied to the Study of Reaction of Epoxy Systems}

Abstracts: The cure reaction of epoxy resin (EP) and curing agents based on polymercaptans (SH), amine-phenol and modified amine was studied in the MIR and NIR spectral regions. It was observed that the FT-NIR shows better the spectrometric changes of the reactions studied, which makes it possible to detect the curing agent in lower contents in epoxide systems.

Keywords: Epoxy resin, curing agent, mercaptans, amine-phenol, modified amine, spectrometric changes, MIR, NIR.

\section{Introdução}

Como é conhecido, as polireações que os grupos 1,2 epóxido conduzidas com a adição de agentes de cura são denominadas reações de cura em resina epoxídica. Os agentes de cura mais conhecidos que promovem estas reações, são as aminas, amidas e anidridos, entretanto mercaptanas e outros agentes de última geração têm sido utilizados neste processo $^{[1,2]}$. Basicamente, as resinas não curadas, que são líquidos altamente viscosos, solúveis em solventes orgânicos, tornam-se materiais rígidos, insolúveis e infusíveis, devido à sua estrutura com ligações cruzadas, e são comumente referidas como resinas epoxídicas ${ }^{[3]}$, apresentando amplo espectro de propriedades viscoelásticas,

As propriedades de um sistema epoxídico dependem do tipo de resina epoxídica, agente de cura, temperatura de cura e do grau de ligação cruzada da reação de cura ${ }^{[4]}$. O entendimento dos grupos formados na reação é essencial para estabelecer a relação de estrutura-propriedade e otimizar as condições de utilização como um adesivo, revestimento, ou matriz em compósitos de fibra-reforçada. A aplicação de técnicas de caracterização se faz necessária para tal fim. Técnicas de análise térmica e espectroscopia no infravermelho (IR), nas regiões MIR e NIR têm sido citadas na literatura ${ }^{[3-7]}$ para avaliação de reações de cura em resinas epoxídicas.

Cherdoud et $\mathrm{al}^{[4]} \mathrm{em}$ seu trabalho, teceram várias considerações sobre as técnicas de caracterização utilizadas para acompanhar mecanismo e cinética de cura, em sistemas epoxídicos. Citaram que técnicas de DSC, nos modos isotérmico e dinâmico, têm sido bastante usadas para explicar a relação de proporcionalidade entre o calor liberado durante a cura e o grau de ligação cruzada, e que, em geral, reações de cura mostram cinética complexa caracterizadas por diversos estágios: indução, geleificação e endurecimento. É necessário obter parâmetros capazes de descrever a cinética do sistema. Caracterização química permanece limitada no estágio inicial da reação, onde a conversão é muito baixa. Métodos espectroscópicos, particularmente, FT-IR, são eficientes para acompanhamento das mudanças químicas durante o processo de cura mesmo em estágio avançado de cura.

Ooi et $\mathrm{al}^{[5]}$ estudaram, por meio de análise DSC, os mecanismos de cura da reação entre resina epoxídica, à base de diglicidil éter de bisfenol A, e agentes imidazóis. Basicamente, verificaram que, entre os agentes usados, 1-metilimidazol (1-MI), 2-metilimidazol (2-MI), 2-fenilimidazol (2-PhI) e 1,2-dimetilimidazol (1,2-DMI),

Autor para correspondência: Rita C. L. Dutra, Divisão de Química, IAE, CTA, Praça Marechal do Ar Eduardo Gomes 50 - Vila das Acácias, CEP: 12228-904, São José dos Campos, SP. E-mail: ritad@iae.cta.br 
o primeiro mostrou-se mais efetivo como agente, resultando em alto grau de conversão e alta $\mathrm{Tg}$, mesmo à baixas concentrações $(2 \% \mathrm{p} / \mathrm{p})$, segundo os autores, evidenciado na sua curva térmica DSC com uma única exoterma.

Os picos observados nas curvas térmicas DSC dos demais agentes imidazóis foram atribuídos à formação de aduto, eterificação (via ânion alcóxido) e a um processo de regeneração de imidazol.

Os autore ${ }^{[5]}$ estudaram o comportamento térmico em DSC dos materiais com e sem isoterma, e verificaram que o grau de conversão de cura de 1-MI foi maior com a utilização de isoterma. Este resultado sugeriu que o comportamento de cura, e então as propriedades, podem ser influenciadas pelas condições de cura, no caso, o programa de temperatura utilizado.

Características, vantagens e desvantagens de técnicas MIR e NIR são discutidas na literatura ${ }^{[3,6,7]}$ para estudo de sistemas epoxídicos. Desde que a principal diferença entre espectros MIR de resinas epoxídicas curadas e não curadas é a ausência da banda do grupo epóxido terminal, em $917 \mathrm{~cm}^{-1}$, e a presença de novas bandas do agente de cura, o processo de cura em resinas epoxídicas pode ser acompanhado por MIR, avaliando-se a intensidade da banda em $917 \mathrm{~cm}^{-1}$. Na região NIR, bandas na região de 4545 e $8628 \mathrm{~cm}^{-1}$ são associadas com o grupo epóxido ${ }^{[3]}$.

Mijovic et al ${ }^{[8]}$ citaram como vantagem da espectroscopia NIR em relação à região MIR, o fato da freqüência NIR poder ser transmitida através de fibras óticas à base de sílica, que são de baixo custo e adequadas em vários tipos e formas.

Poisson et al ${ }^{[7]}$ usaram NIR e MIR para estudar a reação de cura de uma formulação baseada em diglicidiléter de bisfenol A (DGEBA) curada com endurecedor dicianodiamida (DDA). A conversão do grupo epóxido, calculado por NIR (banda de $4530 \mathrm{~cm}^{-1}$ ), foi comparada com os dados obtidos por cromatografia de exclusão por tamanho (SEC) e titulação química como métodos de referência e com MIR, usando a banda $915 \mathrm{~cm}^{-1}$ e a banda de $830 \mathrm{~cm}^{-1}$ (grupo fenil). Uma boa correlação foi encontrada entre SEC, titulação química, e espectroscopia NIR, mas os dados MIR não apresentaram boa concordância, o que foi atribuído ao uso de banda em 915 $\mathrm{cm}^{-1}$. Entretanto, concluíram que estas observações não podem ser generalizadas para todos os sistemas epóxi/amina.

Segundo Weyer ${ }^{[9]}$, o acompanhamento do estudo de reação de cura em resina epoxídica por meio da banda em 4545 $\mathrm{cm}^{-1}$ na região NIR mostra menos interferência do que a vibração de deformação do anel epóxido na região MIR, além da possibilidade da reação poder ser acompanhada com teor mais baixo de grupos epóxido.

Diante da influência dos agentes de cura e das reações por eles promovidas nas propriedades dos sistemas epoxídicos, vantagens e desvantagens apresentadas pelas diferentes técnicas, e a disponibilidade de análise MIR e NIR no mesmo espectrômetro disponível em nossos laboratórios, imaginouse que seria atrativo um estudo qualitativo MIR/NIR para verificação de alterações espectrométricas ocorridas durante as reações de cura em diferentes sistemas epoxídicos.

\section{Experimental}

\section{Materiais}

Amostras de resina EP à base de diglicidiléter de bisfenolA (DGEBA) GENOPOXY 190, em proporções e condições de tempo e $T$ adequadas, foram misturadas, com os agentes de cura (AC) do tipo mercaptana (CAPCURE 3-800), CAPCURE 3-800 e CAPCURE EH-30 (amino-fenol) ou aduto de amina modificada (Versamine CEX 13320), constituindo misturas reacionais EP/CAPCURE 3-800 (1:1), EP/ CAPCURE 3-800/CAPCURE EH-30 (1: 1: 0,1) e EP/ VERSAMINE CEX 13320 (100:40). A temperatura final de cura para EP/CAPCURE 3-800 (1:1) foi de $122{ }^{\circ} \mathrm{C}$ e a amostragem para análise IR foi efetuada à t inicial, 10, 20, $30 \mathrm{~min}, 1 \mathrm{~h}, 2 \mathrm{~h}, 3$ e 4 h), EP/CAPCURE 3-800/CAPCURE EH-30 (1: 1: 0,1) à $120^{\circ} \mathrm{C}$, t inicial, $5,10,15$ e $\left.30 \mathrm{~min}\right)$ e EP/ VERSAMINE CEX 13320 (100:40) à $90^{\circ} \mathrm{C}$, t inicial, 15, 25, 35 min e 1h). Estes materiais foram gentilmente cedidos pela COGNIS S. A. As estruturas químicas dos agentes de cura foram incluídas em trabalhos anteriores ${ }^{[10,11]}$. Para escolha dos tempos, adotou-se um intervalo de análise menor na primeira meia hora de reação, sendo assumido como tempo final de cura, o relacionado com o desaparecimento das bandas analíticas.

\section{Análise por FT-IR de EP, AC e suas misturas reacionais}

Para a obtenção dos espectros foi utilizado o FT-IR SPECTRUM 2000 PERKINELMER com as seguintes condições: região espectral 4000 a $400 \mathrm{~cm}^{-1}$ (MIR) e 6500 a 2000 $\mathrm{cm}^{-1}$ (região espectral parcial NIR), resolução $4 \mathrm{~cm}^{-1}$, ganho 1 e 40 varreduras. Amostras de resina EP e AC foram analisadas na região MIR, puras e como mistura reacional, sendo preparadas como filmes líquidos, curados em estufa, em proporções, à temperatura e tempo já especificados. Para a análise NIR, as amostras foram preparadas como filmes líquidos (amostras puras e como mistura reacional) com o uso de lâminas de vidro nas mesmas condições utilizadas para a análise MIR.

\section{Resultados e Discussão}

Considerando-se que as bandas da resina epoxídica tipo DGEBA são extensivamente discutidas na literatura ${ }^{[3]}$, e as relativas aos $\mathrm{AC}$, já foram discutidas em trabalhos anteriores $^{[10-11]}$, neste trabalho somente serão avaliadas as alterações espectrométricas ocorridas durante a reação de cura, acompanhada nas regiões MIR e NIR.

\section{Análise MIR de EP, CAPCURE-3800 e de sua mistura reacional}

A Figura 1 inclui os espectros da resina epoxídica, CAPCURE 3-800, mistura reacional inicial e após intervalos determinados de tempos de reação. Há um deslocamento do $v$ SH em $2559 \mathrm{~cm}^{-1}$ (intensidade fraca) do agente de cura para $2567 \mathrm{~cm}^{-1}$, no espectro da mistura reacional, absorção que desaparece com 10 minutos de reação, sugerindo rápido 


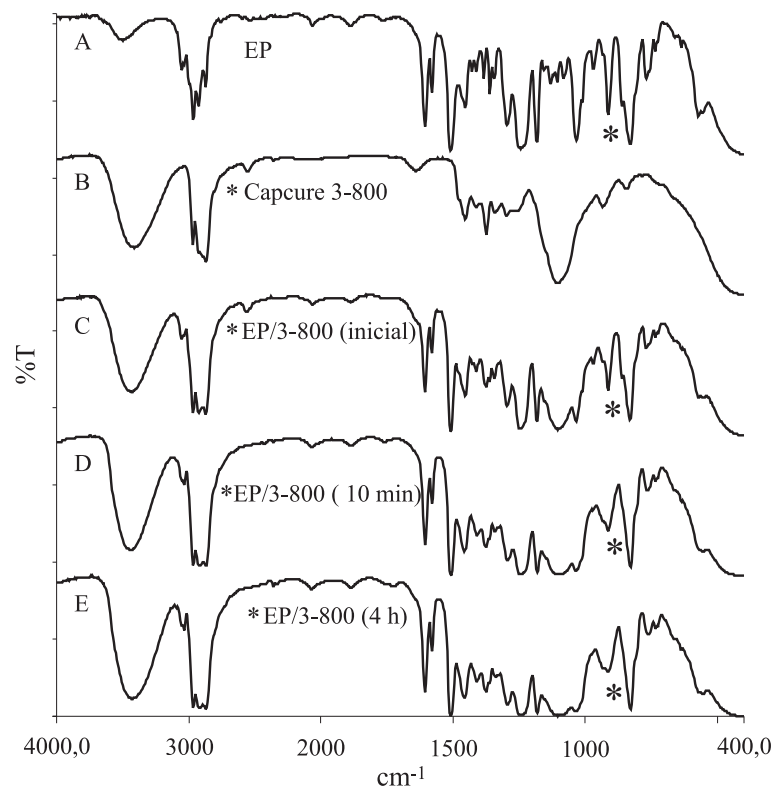

Figura 1. Espectros FT-MIR de EP (A), CAPCURE 3-800 (B), mistura reacional inicial (C), após 10 min (D) e após 4 horas (E).

consumo de grupo e mudança de estrutura. Entretanto, não é possível evidenciar a formação de ligação ${ }^{[1,12]} \mathrm{C}-\mathrm{S}$ entre $700 \mathrm{e}$ $550 \mathrm{~cm}^{-1}$. A região de impressão digital dos espectros dos tempos de reação mostra similaridade com as bandas da resina epoxídica não curada, acrescida de banda do agente em 1106 $\mathrm{cm}^{-1}$ ( $\left.v \mathrm{C}-\mathrm{O}, v \mathrm{~S}-\mathrm{S}\right)$. Pode ser observado na Figura 1, que, basicamente, a única alteração FT-MIR notada é a associada

Tabela 1. Atribuição proposta para as bandas NIR da resina epoxídica ${ }^{[7,13]}$

\begin{tabular}{|c|c|}
\hline $\begin{array}{l}\text { Número de onda } \\
\qquad\left(\mathrm{cm}^{-1}\right)\end{array}$ & Atribuição proposta das bandas \\
\hline 6071 & $\begin{array}{l}\text { Primeiro sobretom da vibração da deformação axial } \\
\text { do grupo terminal metileno (anel oxirano) }\end{array}$ \\
\hline 5986 & $\begin{array}{l}\text { Banda de sobretom da deformação axial do grupo } \\
\text { fenil C-H }\end{array}$ \\
\hline 5886 & Banda de combinação do grupo C-H aromático \\
\hline 5245 & Banda de combinação de grupos $\mathrm{CH}$ e $\mathrm{CH}_{2}$ \\
\hline 4680 & $\begin{array}{l}\text { Banda de combinação da deformação axial do grupo } \\
\mathrm{C}=\mathrm{C} \text { aromático conjugado }\left(\sim 1625 \mathrm{~cm}^{-1}\right) \text { com } \\
\text { deformação axial do grupo } \mathrm{C}-\mathrm{H} 3050 \mathrm{~cm}^{-1}\end{array}$ \\
\hline 4622 & $\begin{array}{l}\text { Banda de combinação da deformação axial do grupo } \\
\mathrm{C}=\mathrm{C} \text { aromático conjugado }\left(1625 \mathrm{~cm}^{-1}\right) \operatorname{com} \\
\text { deformação axial do grupo } \mathrm{C}-\mathrm{H}\left(\sim 3050 \mathrm{~cm}^{-1}\right)\end{array}$ \\
\hline 4530 & $\begin{array}{l}\text { Banda de deformação de } \mathrm{CH}_{2}\left(1460 \mathrm{~cm}^{-1}\right) \\
\text { conjugado com deformação axial do grupo } \mathrm{CH} \\
\text { aromático }\left(\sim 3050 \mathrm{~cm}^{-1}\right) \text { (anel oxirano)/Banda de } \\
\text { combinação do segundo sobretom do estiramento } \\
\text { do anel epoxídico a } 916 \mathrm{~cm}^{-1} \text { com o estiramaento } \\
\left.\text { de } \mathrm{C}-\mathrm{H} \text { à } 2725 \mathrm{~cm}^{-1}\right)\end{array}$ \\
\hline 4480 & Banda de combinação de grupos $\mathrm{CH}_{2}$ e $\mathrm{CH}$ \\
\hline 4351 & Banda de combinação do grupo $\mathrm{CH}_{2}$ \\
\hline 4159 & Banda de combinação do grupo $\mathrm{CH}$ aromático \\
\hline 4065 & Banda de combinação aromática \\
\hline
\end{tabular}

ao grupo $\mathrm{S}-\mathrm{H}$, e que após 4 horas de reação a banda em $917 \mathrm{~cm}^{-1}$ ainda permanece, sugerindo que a resina não está totalmente curada.

\section{Análise NIR de EP, CAPCURE-3800 e de sua mistura reacional}

A Tabela 1 e a Figura 2 mostram as bandas NIR da resina epoxídica, atribuídas aos seus modos vibracionais, de acordo com Poisson ${ }^{[7]}$. Com relação à análise FT-NIR do AC utilizado na mistura reacional, sabe-se que o primeiro sobretom da banda fraca, em $2654 \mathrm{~cm}^{-1}$, do $v$ S-H de mercaptanas ${ }^{[14]}$ como CAPCURE 3-800, ocorre entre 5076 e $5059 \mathrm{~cm}^{-1}$.

As principais alterações espectrométricas FT-NIR observadas para a reação entre EP/CAPCURE 3-800, nos diferentes tempos, estão incluídas na Tabela 2 e Figura 3. Na Tabela 2, as bandas da mistura reacional e as alterações estão assinaladas em negrito. As bandas da mistura reacional inicial estão associadas à presença das bandas da EP e CAPCURE 3-800, e as relativas aos outros tempos de reação, aos deslocamentos em relação às bandas da mistura inicial. Só foram considerados deslocamentos acima de $1 \mathrm{~cm}^{-1}$, por estarem acima da incerteza da medição do espectrômetro, e foram assumidos como deslocamentos máximos, os relativos aos espectros com maiores tempos de reação. O mesmo procedimento foi adotado para os outros sistemas epoxídicos.

De acordo com Musto ${ }^{[13]}$, bandas em $\sim 6070$ e $4530 \mathrm{~cm}^{-1}$ são atribuídas ao anel oxirano. A absorção de maior número de onda é devida ao primeiro sobretom do estiramento do grupo terminal $\mathrm{CH}_{2}$, enquanto a banda de menor número de onda tem sido atribuída à banda de combinação do segundo sobretom do estiramento do anel epóxido à $916 \mathrm{~cm}^{-1} \mathrm{com}$ o estiramento do grupo C-H em torno de $2725 \mathrm{~cm}^{-1}$.
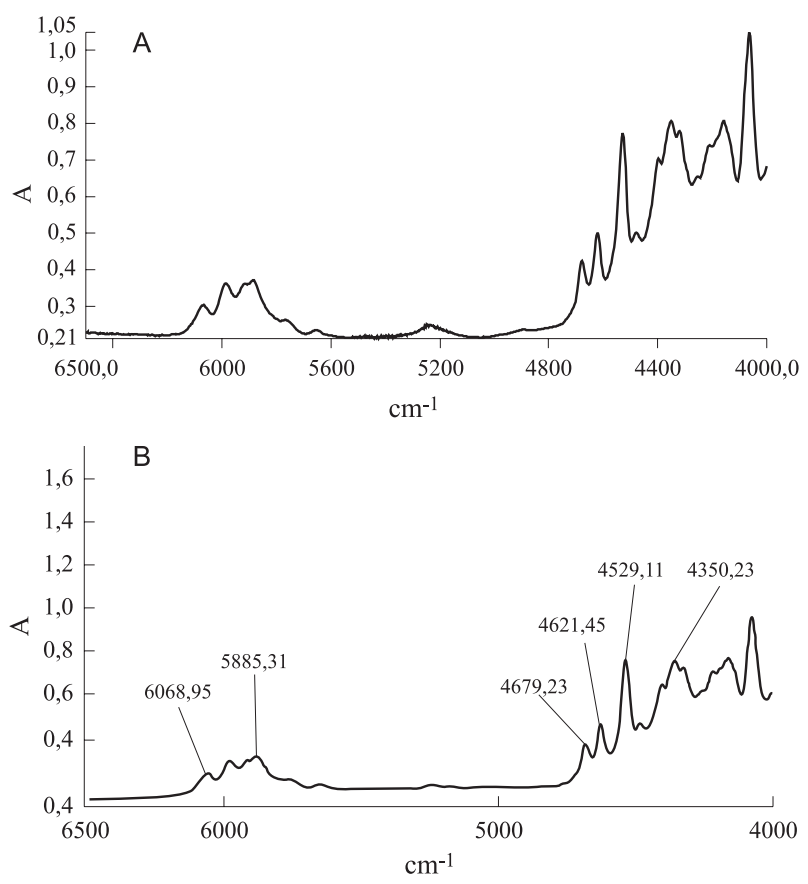

Figura 2. Espectro FT-NIR (6500 a $\left.4000 \mathrm{~cm}^{-1}\right)$ da resina epoxídica utilizada (A) em comparação com a literatura(B $)^{[7]}$ 
Tabela 2. Principais alterações espectrométricas FT-NIR observadas para a reação entre EP/CAPCURE 3-800

\begin{tabular}{|c|c|c|c|c|c|c|c|c|c|}
\hline $\begin{array}{c}\text { Bandas de } \\
\text { CAPCURE } \\
\text { 3-800 } \\
\left(\mathrm{cm}^{-1}\right)\end{array}$ & $\begin{array}{c}\text { Bandas de } \\
\text { EP } \\
\left(\mathbf{c m}^{-1}\right)\end{array}$ & $\begin{array}{c}\text { Bandas da } \\
\text { mistura } \\
\text { reacional } \\
\text { EP/ } \\
\text { CAPCURE } \\
\text { 3-800 } \\
\text { (inicial) }\end{array}$ & $\begin{array}{l}\text { Bandas de } \\
\text { EP/ } \\
\text { CAPCURE } \\
\text { (10 min) }\end{array}$ & $\begin{array}{l}\text { Bandas de } \\
\text { EP/ } \\
\text { CAPCURE } \\
20 \text { min }\end{array}$ & $\begin{array}{c}\text { Bandas de } \\
\text { EP/ } \\
\text { CAPCURE } \\
\text { 30 min }\end{array}$ & $\begin{array}{c}\text { Bandas de } \\
\text { EP/ } \\
\text { CAPCURE } \\
1 \mathrm{~h}\end{array}$ & $\begin{array}{c}\text { Bandas de } \\
\text { EP/ } \\
\text { CAPCURE } \\
2 \text { h }\end{array}$ & $\begin{array}{c}\text { Bandas de } \\
\text { EP/ } \\
\text { CAPCURE } \\
3 \text { h }\end{array}$ & $\begin{array}{c}\text { Bandas de } \\
\text { EP/ } \\
\text { CAPCURE } \\
4 \mathrm{~h}\end{array}$ \\
\hline 5958 & 6071 & 6071 & 6071 & 6070 & - & - & - & - & - \\
\hline- & 5986 & - & - & - & - & - & - & - & - \\
\hline- & 5886 & 5891 & 5891 & 5891 & 5890 & 5890 & 5890 & 5891 & 5891 \\
\hline 5766 & - & 5767 & 5767 & 5766 & 5766 & 5765 & 5762 & 5762 & 5765 \\
\hline 5209 & - & 5209 & 5227 & - & - & - & - & - & - \\
\hline 4780 & 5245 & - & - & 5249 & 5249 & 5249 & 5249 & 5249 & 5249 \\
\hline 4342 & 4680 & 4681 & 4680 & 4679 & 4679 & 4678 & 4678 & 4678 & 4679 \\
\hline 4010 & 4622 & 4623 & 4622 & 4621 & 4621 & 4620 & 4620 & 4620 & 4620 \\
\hline- & 4530 & 4529 & 4529 & 4528 & 4529 & 4528 & 4527 & 4527 & 4528 \\
\hline- & 4480 & - & - & - & - & - & - & - & - \\
\hline- & 4351 & 4346 & 4346 & 4345 & 4344 & 4344 & 4344 & 4344 & 4344 \\
\hline- & 4159 & 4157 & 4156 & 4157 & 4161 & 4161 & 4161 & 4161 & 4161 \\
\hline- & 4065 & 4062 & 4062 & 4061 & 4060 & 4060 & 4060 & 4060 & 4060 \\
\hline
\end{tabular}

Nesta reação, a banda em $6071 \mathrm{~cm}^{-1}$ da resina desaparece após 20 min e a de $4530 \mathrm{~cm}^{-1}$ mostra pequeno deslocamento para $4527 \mathrm{~cm}^{-1}$. Estas alterações associadas ao deslocamento de bandas do agente acima de $5000 \mathrm{~cm}^{-1}$, sugerem que a resi-

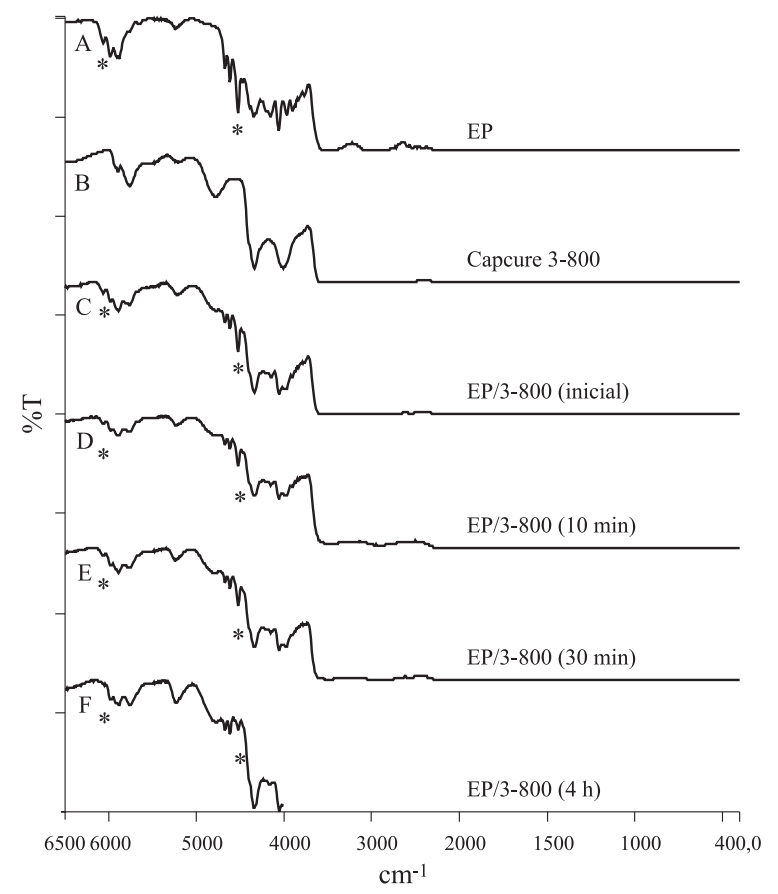

Figura 3. Espectros parciais (6500 a $\left.4000 \mathrm{~cm}^{-1}\right)$ FT-NIR de EP (A), CAPCURE 3-800 (B), mistura reacional inicial (C), após 10 min (D), 30 $\min (\mathrm{E}), 4$ horas $(\mathrm{F})$. na está sendo submetida a um processo de cura, que entretanto, não termina até 4 horas de reação, pois não é observado desaparecimento de todas as bandas analíticas, da mesma forma que foi mostrado na análise MIR do sistema.

Basicamente, as alterações espectrométricas observadas nesta reação entre resina epoxídica e CAPCURE 3-800, acompanhada na região NIR, sugerem algum consumo dos grupos oxirano da resina e SH do agente, mudanças de vizinhanças de grupos $\mathrm{CH}$ aromáticos da resina, como esperado. Observou-se, também, que as alterações para este sistema na região NIR são mais nítidas que as assinaladas na região MIR.

Segundo a literatura ${ }^{[1]}$, é necessária a adição de uma amina terciária para melhorar a reatividade dos mercaptans. De acordo com Cui ${ }^{[2]}$, a reação do grupo mercaptan, de um agente contendo grupos amina terciária, e epóxido foi similar à aquela observada para o grupo $\mathrm{OH}$, embora, como o poder nucleofílico do enxofre é maior do que o oxigênio, a mercaptana curou à temperatura ambiente na presença de acelerador.

Grupos $\mathrm{OH}$ reagem com epoxídicos, catalisados por ácido, produzindo uma ligação tipo éter ${ }^{[15]}$ :<smiles>CC(C)O[C@H]1O[C@@H]1CC(C)(C)OCCO</smiles>

Analogamente para os agentes contendo SH, tem-se a formação de tio-éter 
<smiles>CC(C)[SnH2]C1O[C@H]1C(C)(C)SCC(O)O</smiles>

Estes agentes contendo SH são extremamente reativos devido à formação de íon mercáptido, que é criado na presença de uma resina epoxídica e amina terciária.<smiles>[R]N([R])[R]N([R])[R]</smiles>

O íon mercáptido é formado quando a mercaptana doa seu hidrogênio. Esta espécie química tem uma alta velocidade de deslocamento nucleofílico. Desloca a amina terciária para formar ligação covalente carbono-enxofre. A amina terciária é regenerada e é livre para trabalhar novamente sobre outro sítio oxirano.

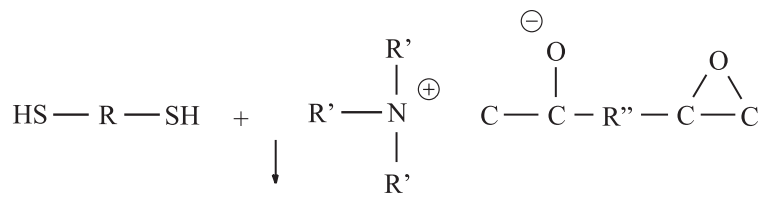

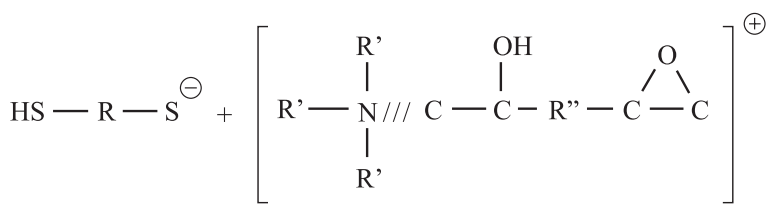

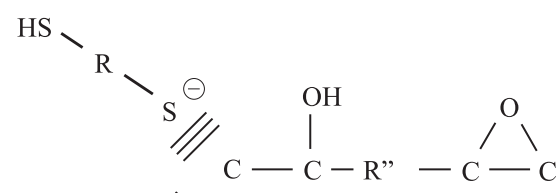

$$
\begin{aligned}
& \mathrm{R}^{\mathrm{R}}-\mathrm{N}_{\mathrm{N}}^{\mathrm{R}} \\
& \mathrm{HS}-\mathrm{R}-\mathrm{S}-\mathrm{C}-\mathrm{C}-\mathrm{R}=\mathrm{OH}-\mathrm{C}+\stackrel{\mathrm{O}}{\stackrel{\mathrm{O}}{\mathrm{N}} \mathrm{R}_{\mathrm{R}}^{\prime}}
\end{aligned}
$$

Visando avaliar a cura da resina epoxídica contendo mercaptana em presença de amina terciária ${ }^{[1,10]}$, CAPCURE EH-30 foi adicionado ao sistema epoxídico EP/CAPCURE $3-800$.

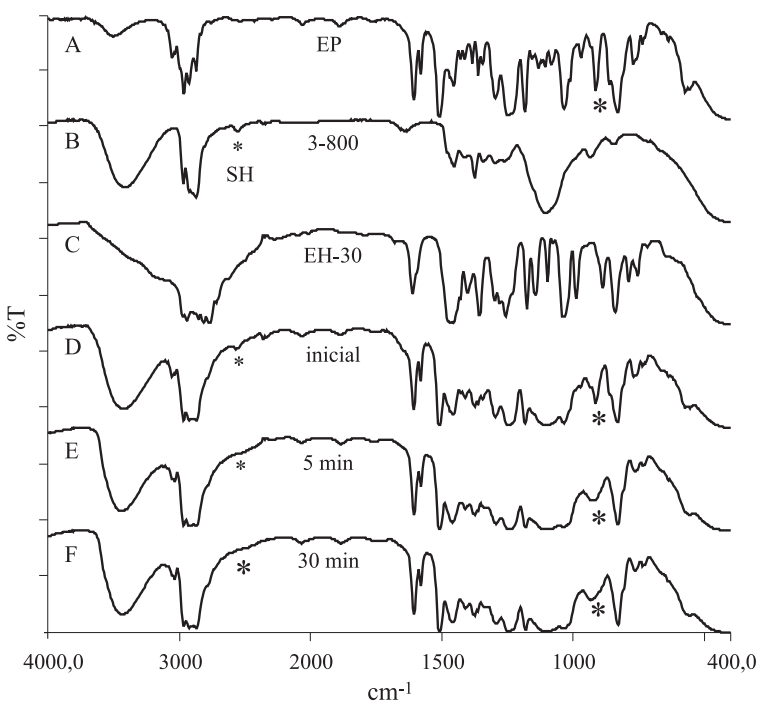

Figura 4. Espectros FT-MIR de EP (A), CAPCURE 3-800 (B), CAPCURE EH-30 (C), mistura reacional inicial (D), após $5 \mathrm{~min}(\mathrm{E}), 30 \mathrm{~min}(\mathrm{~F})$

\section{Análise FT- MIR de EP, CAPCURE-3800 e CAPCURE EH-30 e de sua mistura reacional}

A Figura 4 inclui os espectros FT-MIR da resina epoxídica, CAPCURE 3-800, CAPCURE EH-30, mistura reacional inicial e após determinados tempos de reação. Há formação de banda em $\sim 3440 \mathrm{~cm}^{-1}$ ( $\left.v \mathrm{OH}\right)$ e um deslocamento do $v \mathrm{SH}$ em $2559 \mathrm{~cm}^{-1}$ do agente de cura para $2570 \mathrm{~cm}^{-1}$ no espectro da mistura reacional, absorção que desaparece com 05 minutos de reação. O mesmo acontecendo, neste período, com a banda da resina em $915 \mathrm{~cm}^{-1}$ da resina, dando lugar a uma banda em $932 \mathrm{~cm}^{-1}$, que pode, provavelmente ser atribuída à deformação de grupo ${ }^{[16]} \mathrm{OH}$ formado na cura. A diferença entre a cura deste sistema e o contendo somente CAPCURE 3-800 é que a presença da amina terciária, CAPCURE EH30 diminui o tempo de reação, como esperado ${ }^{[1]}$.

\section{Análise FT-NIR de EP, CAPCURE-3800 e CAPCURE EH-30 e de sua mistura reacional}

Com relação à análise FT-NIR do CAPCURE EH-30, que contém grupos $\mathrm{OH}$ fenólicos e $\mathrm{C}-\mathrm{H}$ aromáticos, sabe-se que vibrações de estiramento dos primeiros grupos ${ }^{[9]}$ apresentam bandas de combinação próximo de $5000 \mathrm{~cm}^{-1}$, e C-H aromáticos mostram o primeiro sobretom em torno de $5930 \mathrm{~cm}^{-1} \mathrm{e}$ bandas de combinação entre 4650 e $4065 \mathrm{~cm}^{-1}$.

As principais alterações espectrométricas observadas para a reação entre EP/CAPCURE 3-800/CAPCURE EH-30, nos diferentes tempos, estão incluídas na Tabela 3 e Figura 5.

A banda em $6071 \mathrm{~cm}^{-1}$ do anel oxirano não é notada nem após 5 minutos de reação; a absorção em $5766 \mathrm{~cm}^{-1}$, comum aos dois agentes CAPCURE 3-800 e CAPCURE EH-30, provavelmente associadas aos grupos $\mathrm{SH}$ e $\mathrm{C}-\mathrm{H}$ aromático, sofre um deslocamento para $5758 \mathrm{~cm}^{-1}$; a banda em 5209 $\mathrm{cm}^{-1}$ do CAPCURE 3-800, provavelmente, associada ao grupo SH desloca-se para $5228 \mathrm{~cm}^{-1}$.

Basicamente, as alterações espectrométricas observadas nesta reação entre resina epoxídica, CAPCURE 3-800 e 
Tabela 3. Principais alterações espectrométricas FT-NIR observadas para a reação entre EP/CAPCURE 3-800/CAPCURE EH-30

\begin{tabular}{|c|c|c|c|c|c|c|c|}
\hline $\begin{array}{c}\text { Bandas de } \\
\text { CAPCURE } \\
3-800 \\
\left(\mathbf{c m}^{-1}\right)\end{array}$ & $\begin{array}{c}\text { Bandas de } \\
\text { CAPCURE } \\
\text { EH-30 }\end{array}$ & $\begin{array}{r}\text { Bandas } \\
\text { de EP } \\
\left(\mathrm{cm}^{-1}\right)\end{array}$ & $\begin{array}{c}\text { Bandas da } \\
\text { mistura } \\
\text { reacional } \\
\text { EP/CAPCURE } \\
\text { 3-800/ } \\
\text { CAPCURE } \\
\text { EH-30 } \\
\text { (inicial) }\end{array}$ & $\begin{array}{c}\text { Bandas de } \\
\text { EP/CAPCURE } \\
\text { 3-800/ } \\
\text { CAPCURE } \\
\text { EH-30 } \\
\text { (5 min) }\end{array}$ & $\begin{array}{c}\text { Bandas de } \\
\text { EP/CAPCURE } \\
\text { 3-800/ } \\
\text { CAPCURE } \\
\text { EH-30 } \\
10 \mathrm{~min}\end{array}$ & $\begin{array}{c}\text { Bandas de } \\
\text { EP/CAPCURE } \\
\text { 3-800/ } \\
\text { CAPCURE } \\
\text { EH-30 } \\
15 \text { min }\end{array}$ & $\begin{array}{c}\text { Bandas de } \\
\text { EP/CAPCURE } \\
\text { 3-800/ } \\
\text { CAPCURE } \\
\text { EH-30 } \\
\text { 30 min }\end{array}$ \\
\hline 5958 & 5927 & 6071 & 6066 & - & - & - & - \\
\hline - & - & 5986 & - & - & - & - & - \\
\hline- & - & 5886 & 5893 & 5888 & 5891 & 5894 & 5892 \\
\hline 5766 & 5769 & - & 5767 & 5762 & 5764 & 5766 & 5758 \\
\hline 5209 & 5226 & - & 5228 & 5228 & - & - & - \\
\hline 4780 & 4622 & 5245 & 5245 & - & 5244 & 5240 & 5239 \\
\hline 4342 & 4375 & 4680 & 4681 & 4679 & 4677 & 4676 & 4676 \\
\hline 4010 & 4206 & 4622 & 4622 & 4621 & 4620 & 4620 & 4620 \\
\hline - & 4021 & 4530 & 4529 & - & - & - & - \\
\hline - & 4136 & 4480 & - & - & - & - & - \\
\hline- & 4021 & 4351 & 4346 & 4345 & 4344 & 4343 & 4344 \\
\hline- & - & 4159 & 4157 & - & - & - & - \\
\hline
\end{tabular}

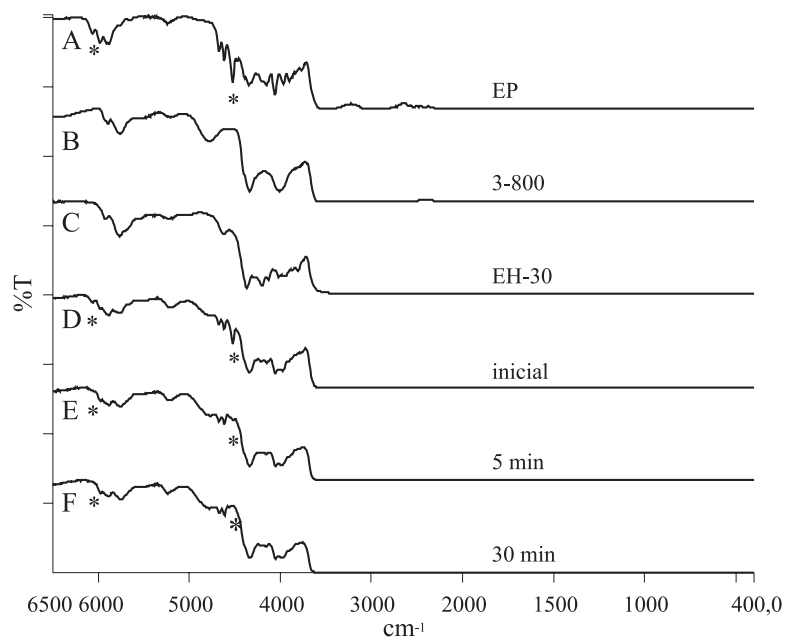

Figura 5. Espectros parciais (6500 a $\left.4000 \mathrm{~cm}^{-1}\right)$ FT-NIR de EP (A), CAPCURE 3-800 (B), CAPCURE EH-30 (C), mistura reacional inicial (D), após $5 \min (\mathrm{E}), 30 \mathrm{~min}(\mathrm{~F})$.

CAPCURE EH-30, acompanhada na região NIR, sugerem alterações nos grupos oxirano e $\mathrm{CH}$ aromáticos da resina, SH do agente mercaptan, como esperado.

É interessante destacar que a presença de CAPCURE EH30, mesmo em pequena proporção, é observada na análise NIR (banda em $5228 \mathrm{~cm}^{-1}$ ) e não na MIR.
Análise FT- MIR da mistura reacional EP/VERSAMINE CEX 13320

A Figura 6 inclui os espectros da resina epoxídica, Versamine CEX 13320, mistura reacional inicial e após intervalos determinados de tempos de reação. $\mathrm{O}$ espectro da mistura reacional apresenta, basicamente, absorções da resina epoxídica e dos grupos amínicos do agente de cura. Os espectros que representam as absorções dos tempos 15, 25, 35 min e 1 hora mostram que, basicamente, aparece uma banda em $3411 \mathrm{~cm}^{-1}$, provavelmente atribuída ao $\mathrm{vOH}$. As bandas da resina em 1157, 1131 e $1011 \mathrm{~cm}^{-1}$, região de $v \mathrm{C}-\mathrm{O}$, são deslocadas para 1148, 1107, $1084 \mathrm{e} 1037 \mathrm{~cm}^{-1}$. A banda da resina em $772 \mathrm{~cm}^{-1}$ ( $\delta \mathrm{CH}$ aromático) desaparece, observando-se uma em $755 \mathrm{~cm}^{-1}$ sugerindo mudança relacionada à substituição aromática.

\section{Análise NIR de EP e VERSAMINE CEX 13320 e de sua mistura reacional}

Com relação à análise FT-NIR do VERSAMINE CEX 13320, um aduto de amina, que contém grupos $\mathrm{OH}$ fenólicos e C-H aromáticos, sabe-se que vibrações de estiramento dos primeiros grupos ${ }^{[9]}$ apresentam bandas de combinação próximo de $5000 \mathrm{~cm}^{-1}$, e C-H aromáticos mostram o primeiro sobretom em torno de $5930 \mathrm{~cm}^{-1}$ e bandas de combinação entre 4650 e $4065 \mathrm{~cm}^{-1}$. 


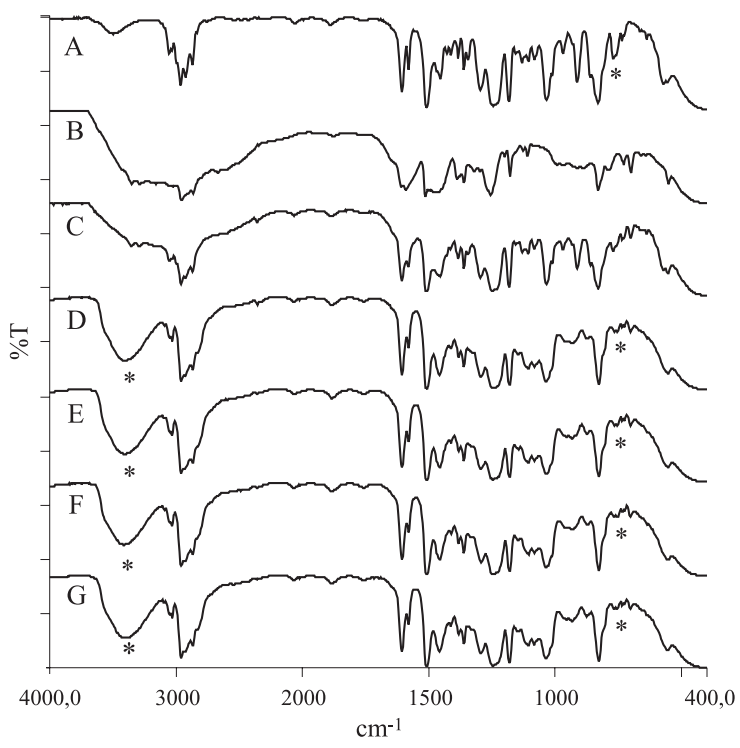

Figura 6. Espectros FT-MIR de EP (A), VERSAMINE CEX 13320 (B), mistura reacional inicial (C), após $15 \mathrm{~min}(\mathrm{D}), 25 \mathrm{~min}(\mathrm{E}), 35 \mathrm{~min}(\mathrm{~F}) \mathrm{e}$ 1h (G).

As principais alterações espectrométricas NIR observadas para a reação entre EP/VERSAMINE CEX 13320, nos diferentes tempos, estão incluídas na Tabela 4 e Figura 7.

Basicamente, observa-se que as principais alterações espectrométricas consistem no desaparecimento da banda do agente de cura, em torno de $4930 \mathrm{~cm}^{-1}$, provavelmente associada ao $\mathrm{CH}$ aromático, antes de 15 minutos de reação, e da resina, em $4530 \mathrm{~cm}^{-1}$ do anel oxirano, após 35 minutos de reação.

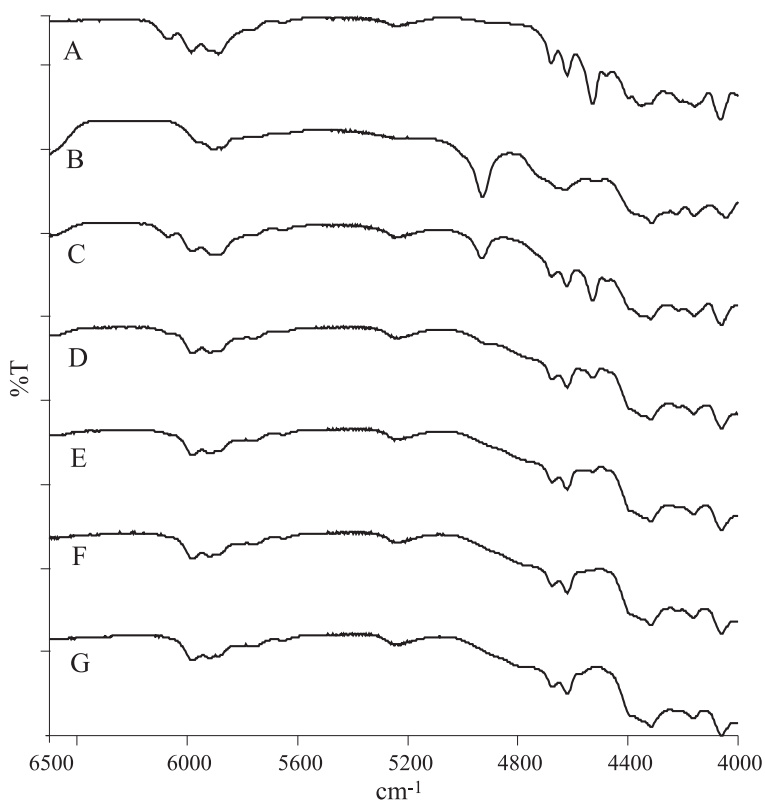

Figura 7. Espectros parciais (6500 a $\left.4000 \mathrm{~cm}^{-1}\right)$ FT-NIR de EP (A), VERSAMINE CEX 13320 (B), mistura reacional inicial (C), após $15 \mathrm{~min}$ (D), $25 \min (\mathrm{E}), 35 \mathrm{~min}(\mathrm{~F})$ e $1 \mathrm{~h}(\mathrm{G})$.

\section{Conclusões}

Uma resina epoxídica, tipo DEGBA, curada com agentes à base de mercaptana e aminas terciárias, foi caracterizada por espectroscopia FT-MIR e FT-NIR com relação, basicamente, ao desaparecimento de grupos funcionais durante a cura. Foi observado que a espectroscopia FT-NIR evidencia

Tabela 4. Principais alterações espectrométricas observadas para a reação entre EP/VERSAMINE CEX 13320

\begin{tabular}{|c|c|c|c|c|c|}
\hline $\begin{array}{c}\text { Bandas de } \\
\text { VERSAMINE } \\
\text { CEX 13320 } \\
\left(\mathrm{cm}^{-1}\right)\end{array}$ & $\begin{array}{c}\text { Bandas de EP } \\
\left(\mathrm{cm}^{-1}\right)\end{array}$ & $\begin{array}{l}\text { Bandas da mistura } \\
\text { reacional } \\
\text { EP/ VERSAMINE } \\
\text { CEX 13320 } \\
\text { (inicial) } \\
\left(\mathrm{cm}^{-1}\right)\end{array}$ & $\begin{array}{c}\text { Bandas da mistura } \\
\text { reacional } \\
\text { EP/VERSAMINE } \\
\text { CEX 13320 } \\
(15 \text { min }) \\
\left(\mathrm{cm}^{-1}\right)\end{array}$ & $\begin{array}{c}\text { Bandas da mistura } \\
\text { reacional } \\
\text { EP/VERSAMINE } \\
\text { CEX 13320 } \\
\left(25 \mathrm{~min}^{-1}\right) \\
\left(\mathrm{cm}^{-1}\right)\end{array}$ & $\begin{array}{c}\text { Bandas da mistura } \\
\text { reacional } \\
\text { EP/VERSAMINE } \\
\text { CEX 13320 } \\
(35 \text { min }) \\
\left(\mathrm{cm}^{-1}\right)\end{array}$ \\
\hline- & 6071 & 6069 & - & - & - \\
\hline - & 5986 & 5985 & 5981 & 5982 & 5981 \\
\hline 5906 & 5886 & 5885 & 5918 & 5921 & 5924 \\
\hline - & - & 5650 & 5649 & 5649 & 5649 \\
\hline 4929 & - & 4932 & - & - & - \\
\hline- & 5245 & - & 5249 & 5249 & 5249 \\
\hline- & 4680 & 4679 & 4677 & 4677 & 4677 \\
\hline 4630 & 4622 & 4623 & 4622 & 4622 & 4622 \\
\hline 4314 & 4530 & 4529 & 4529 & 4530 & - \\
\hline 4228 & 4480 & - & - & - & - \\
\hline 4161 & 4351 & 4160 & 4162 & 4163 & 4163 \\
\hline
\end{tabular}


melhor, que a região MIR, as alterações espectrométricas ocorridas durante as reações estudadas entre resina e agentes de cura, permitindo inclusive detectar, o agente de cura em menor proporção no sistema epoxídico.

\section{Referências Bibliográficas}

1. Catálogo Cognis 2001.

2. Cui, Z.; Lu, C.; Yang, B.; Shen, J.; Su, X.; Yang, H. Poymer 42, p.10095 (2001).

3. Urbanski, J.; Czerwinski, W.; Janicka, K.; Majewska, F.\& Zowall, H. - "Handbook of analysis of synthetic polymers and plastics", John Wiley, New York (1977).

4. Cherdoud-Chihani, A.; Mouzali, M.; Abadie, M. J. M. Journal of Applied Polymer Science 69, p.1167 (1998).

5. Ooi, S. K.; Cook, W. D.; Simon, G. P.; Such, C. H. Polymer 41, p.3639 (2000).

6. Kathryrin, A; Lee, B. - Applied Spetroscopy Rewiews 28, 3, p.231 (1993).

7. Poisson, N.; Lachenal, G.; Sautereau, H. - Vibrational Spectroscopy 12, 237 (1996).
8. Mijovié, J.; Andejelié, S. - Polymer 37, 8, 1295 (1996).

9. Weyer, L. G. - Applied Spectroscopy Reviews 21, 1, 1 (1985).

10. Romão, B. M. V.; Diniz, M. F.; Azevedo, M. F. P.; Lourenço, V. L.; Pardini, L. C.; Dutra, R. C. L.; Burel, F. - Polímeros: Ciência e Tecnologia, 2003, no prelo

11. Romão, B. M. V.; Diniz, M. F.; Azevedo, M. F. P.; Lourenço, V. L.; Pardini, L. C.; Dutra, R. C. L.; Burel, F. Polymer Bulletin, no prelo.

12. Smith, A . L. - "Applied Infrared Spectroscopy", John Wiley, New York (1979).

13. Musto, P.; Mascia, L.; Ragosta, G.; Scaringi, G.; Villano, P. - Polymer 41, 565, (2000).

14. Goddu, R. F.; - Advances in Analytical Chemistry and Instrumentation, 1, 347 (1960).

15. Lee, H; Neville, K; - "Handbook of epoxy resins" Mc Gram Hill, Inc USA (1967).

Enviado: 05/09/03

Reenviado: $10 / 03 / 04$

Aprovado: 10/04/04 